\title{
An explicit topology optimization method using moving polygonal morphable voids (MPMVs)
}

\author{
Van-Nam Hoang*
}

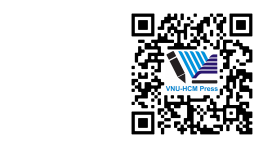

Use your smartphone to scan this QR code and download this article
Mechanical Engineering Institute, Vietnam Maritime University, Hai Phong City, Viet Nam

\section{Correspondence}

Van-Nam Hoang. Mechanical Engineering Institute, Vietnam Maritime University, Hai Phong City, Viet Nam

Email:namhv.vck@imaru.edu.vn

History

- Received: 2020-04-10

- Accepted: 2020-06-12

- Published: 2020-06-27

DOI : 10.32508/stdj.v23i2.2067

\section{Check for updates}

\section{Copyright}

(c) VNU-HCM Press. This is an openaccess article distributed under the terms of the Creative Commons Attribution 4.0 International license.

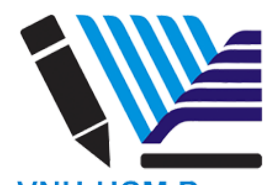

VNU-HCM Press

\begin{abstract}
Introduction: Conventional topology optimization approaches are implemented in an implicit manner with a very large number of design variables, requiring large storage and computation costs. In this study, an explicit topology optimization approach is proposed by moving polygonal morphable voids whose geometry parameters are considered as design variables. Methods: Each polygonal void plays as an empty-material zone that can move, change its shapes, and overlap with its neighbors in a design space. The geometry parameters of MPMVs consisting of the coordinates of polygonal vertices are utilized to render the structure in the design domain in an element density field. The density function of the elements located inside polygonal voids is described by a smooth exponential function that allows utilizing gradient-based optimization solvers. $\mathbf{R e}$ sults \& Conclusion: Compared with conventional topology optimization approaches, the MPMV approach uses fewer design variables, ensure mesh-independence solution without filtering techniques or perimeter constraints. Several numerical examples are solved to validate the efficiency of the MPMV approach.

Key words: Topology optimization, Moving morphable void, Moving morphable bar
\end{abstract}

\section{INTRODUCTION}

Topology optimization is typically described by searching the distribution of a given amount of material in a prescribed design domain to maximize the structural performance, i.e., the structural compliance, buckling load, displacement. Over the past three decades, topology optimization has undergone a long period of development, contributed by researchers around the world. Topology optimization has been integrated into commercial software such as Comsol, Altair, and Ansys as a powerful tool for structural optimization solutions. Until now, the majority of existing approaches have been implicit, that is, the structure is implicitly described by element density fields $\left(\mathrm{SIMP}^{1}, \mathrm{ESO}^{2}\right.$ ) or level-set functions ${ }^{3}$. One of the disadvantages of the implicit approaches is that they have a very large number of design variables, equal to the number of grid elements (or the number of grid nodes) of the design domain. The optimization requires large storage capacity as well as demanding calculations.

To reduce the number of design variables as well as minimize computational costs, explicit topology optimization approaches have been proposed recently ${ }^{4-7}$, in which the structure is explicitly described by geometry parameters of geometric components. The benefits of the explicit approaches can be listed as using fewer design variables, using an explicit structural description that is convenient for the post-processing stage, and straightforward feature size control ${ }^{7} . \operatorname{In}^{7}$, we introduced an explicit topology optimization approach using moving morphable bars for the design of structural compliance and compliant mechanism problems. The extension of the moving morphable bar approach ${ }^{7}$ has been applied in several applications in recent years, i.e., coated designs ${ }^{8}$, embedded components $^{9}$, and cellular structures ${ }^{10}$.

The aforementioned explicit approaches mostly used non-flexible components like circles, bars, and ellipses $^{4-7}$ or complex flexible components using Bsplines/NURBS ${ }^{11,12}$. In this study, we will model simple flexible components using polygonal voids for explicit topology optimization of two-dimensional structures. The structural optimization is performed by optimizing the positions of the vertices of the polygonal voids.

\section{MPMV}

We consider an MPMV with $n_{s}$ connected segments denoted by $\Omega_{k}$ as illustrated in Figure 1. The segment $i$ is determined by the coordinates of two adjacent vertices $\mathbf{x}_{\boldsymbol{i}}$ and $\mathbf{x}_{i+1}$. Let $\mathbf{T}$ be a vector originating from $\mathbf{x}_{i}$ to $\mathbf{x}_{i+1}$, defined by $\mathbf{T}=\mathbf{x}_{i+1}-\mathbf{x}_{i}$. The unit vector $\mathbf{t}$ 


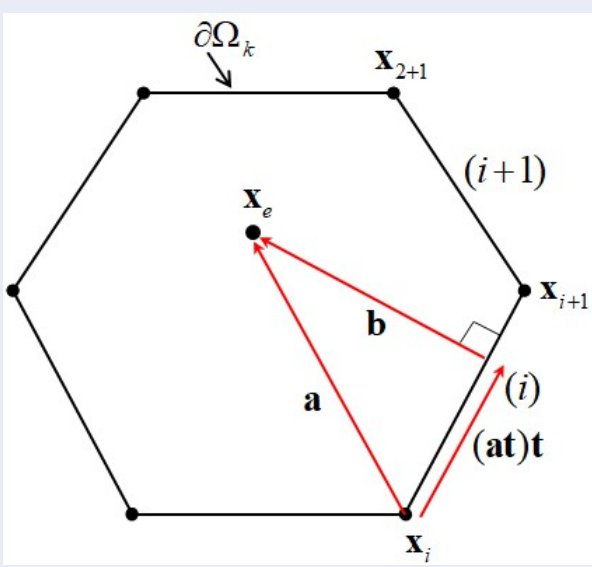

Figure 1: MPMV: an MPMV consists of $\boldsymbol{n}_{s}$ line segments that connect at their vertices of the polygon.

along the line segment $i$ is given by:

$$
t=\frac{\mathbf{T}}{\|\mathbf{T}\|}=\frac{\mathbf{x}_{i+1}-\mathbf{x}_{i}}{\left\|\mathbf{x}_{i+1}-\mathbf{x}_{i}\right\|}
$$

We name $d_{e k}^{(i)}$ as the minimum distance from the center element $\mathbf{x}_{e} \in \Omega_{k}$ to the segment $i$ of the void $k, d_{e k}^{(i)}$ can be expressed by

$d_{e k}^{(i)}=\left\{\begin{array}{l}\|\mathbf{a}\|=\left\|\mathbf{x}_{e}-\mathbf{x}_{i}\right\| \text { if } \mathbf{a t} \leq 0 \\ \|\mathbf{b}\|=\|\mathbf{a}-(\mathbf{a t}) \mathbf{t}\| \text { if } 0<\mathbf{a t}<\mathbf{t} \mathbf{T}\end{array}\right.$

where $\mathbf{a}=\mathbf{x}_{e}-\mathbf{x}_{i}$ is the vector originating from $\mathbf{x}_{i}$ to $\mathbf{x}_{e}$ and $\mathbf{b}=\mathbf{a}-(\mathbf{a t}) \mathbf{t}$ is a perpendicular vector of the segment $i$ with its length equal to the minimum distance from the element $e$ to the line through $\mathbf{x}_{i}$ and $\mathbf{x}_{i+1}$ (see Figure 1).

$$
\begin{gathered}
\rho_{e}= \begin{cases}1 & \forall \mathbf{x}_{e} \notin \bigcup_{k=1}^{n_{v}} \Omega_{k} \\
\prod_{k=1}^{n_{v}} e^{-\beta d_{e k}} & \forall \mathbf{x}_{e} \in \bigcup_{k=1}^{n_{v}} \Omega_{k}\end{cases} \\
d_{e k}=\min \left\{d_{e k}^{(i)}\right\}, i=1,2, \ldots, n_{s},
\end{gathered}
$$

where $d_{e k}$ is the minimum distance from element $e$ to the boundary $\partial \Omega_{k}$ of the void $k ; n_{v}$ is the number of voids and $\beta$ is a positive control number to enforce element density to converge to 0 or 1 (see Figure 2). In equation (3), $\rho_{e}=1$ if the element does not locate inside the void zones (solid material), $\rho_{e}=0$ if the element locates inside the void zones (voids), and $0<$ $\rho_{e}<1$ responds to the elements around the structural boundaries.

It is worth noting that only elements located inside void zones are considered in the calculation of the element densities and their sensitivities. Of course, this will significantly reduce the mapping time compared with the case of geometric mapping components onto the full grid like most of the current explicit approaches. Figure 2 shows plots of function $\varnothing=e^{-\beta d_{e k}}$ with respect to the minimum distance $d_{e k}$ for different values of $\beta$.

The larger $\beta$ results in a narrower band of nonphysical material around the structural boundaries. $\beta$ should be selected so that there is a transition zone (lowdensity element zone) between solid material phase and void phase, to ensure the existence of non-zeros derivation of the element density function for employing gradient-optimization solvers. In this work, the selection $\beta=2$ corresponds to about one lowdensity element on the boundaries for unit-length element mesh.

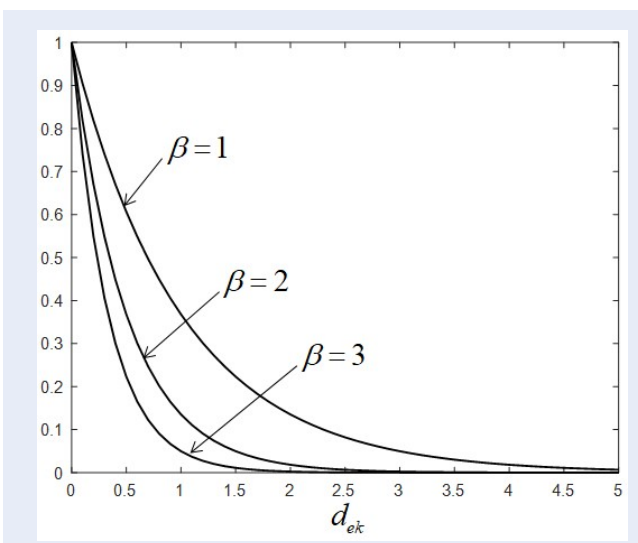

Figure 2: The control parameter $\beta$ : plots of function $\varnothing=e^{-\beta d_{e k}}$ with respect to $d_{e k}$ for different values of $\beta$.

\section{TOPOLOGY OPTIMIZATION USING MPMVS}

The compliance minimal problem is considered in this work. The objective is searching for an optimal set of geometry parameters to minimize structural compliance. The optimization problem is formulated as

$$
\begin{aligned}
& \min _{\mathbf{x}} c(\mathbf{x})=\sum_{e=1}^{n_{e}} \chi \mathbf{u}_{e}^{T} \mathbf{k}_{0} \mathbf{u}_{e} \\
& \text { subject to } \frac{1}{\left|\Omega_{0}\right|} \int_{\Omega_{0}} \rho_{e} d \Omega-f \leq 0 \\
& \mathbf{x}_{\min } \leq \mathbf{x} \leq \mathbf{x}_{\max }
\end{aligned}
$$

where $c$ is the structural compliance; $n_{e}$ is the total number of elements, $\mathbf{k}_{0}$ is the element stiffness matrix; $\mathbf{u}_{e} \subset \mathbf{u}=\mathbf{K}^{-1} \mathbf{F}$ is the element displacement vector; $\mathbf{K}, \mathbf{u}$ and $\mathbf{F}$ are the global stiffness matrix, global displacement vector, and global force vector, respectively; $\left|\Omega_{0}\right|$ denotes the design-domain volume; $f$ denotes the allowed material volume ratios; 
$\mathbf{x}=\left\{\mathbf{x}_{k}\right\}, \mathbf{x}_{k}=\left\{\mathbf{x}_{i}\right\}, i=1,2, \ldots, n_{s}, k=1,2, \ldots, n_{v}$ is the variable vector; $\mathbf{x}_{\min }, \mathbf{x}_{\max }$ are the bounds of $\mathbf{x}$ with a note that it is not necessary to set strict limits for lower and upper bounds of the design variables. This means that the vertex of polygonal voids can move out of the design domain as can be seen in Figure $4 \mathrm{~b}$. The material interpolation in SIMP $^{1}$ is employed,

$$
\chi=\rho_{\min }+\rho_{e}^{\eta}\left(1-\rho_{\min }\right)
$$

where $\rho_{\text {min }}=10^{-4}$ is added to ensure a well-posed finite element analysis.

Sensitivity analysis of the objective function is expressed by the following equation,

$$
\frac{\partial c}{\partial \xi}=\sum_{e=1}^{n_{e}} \frac{\partial c}{\partial \rho_{e}} \frac{\partial p_{e}}{\partial d_{e k}} \frac{\partial d_{e k}}{\partial \xi}
$$

where $\xi \subset \mathbf{x}$ is an arbitrary design variable in the variable vector $\mathbf{x}$, the derivative of the objective function to element density $\partial c / \partial \rho_{e}$ is derived from Eqs. (5-6),

$$
\frac{\partial \rho_{c}}{\partial \rho_{e}}=\eta \rho_{e}^{\eta-1}\left(1-\rho_{\min }\right) \mathbf{u}_{e}^{T} \mathbf{k}_{0} \mathbf{u}_{e},
$$

the derivative of element density function to the minimum distance $\partial \rho_{e} / \partial d_{e k}$ is derived from Equation (3),

$$
\frac{\partial p_{e}}{\partial d_{e k}}=\left\{\begin{array}{l}
0, \forall \mathbf{x}_{e} \notin \bigcup_{k=1}^{n_{v}} \Omega_{k} \\
-\beta \rho_{e}, \forall \mathbf{x}_{e} \in \bigcup_{k=1}^{n_{v}} \Omega_{k}
\end{array}\right.
$$

and to determine $\partial d_{e k} / \partial \xi$, we suppose that the minimum distance function in Equation (4) is readily known, that means, $d_{e k}=d_{e k}^{(i)}$. Derivative expressions of $\partial d_{e k}^{(i)} / \partial \xi$ is derived from Equation (2) as follows,

$$
\frac{\partial d_{e k}^{(i)}}{\partial \mathbf{x}_{i}}=\left\{\begin{array}{l}
-\mathbf{a} \frac{1}{\|\mathbf{a}\|}, \text { if } \mathbf{a t} \leq 0 \\
\left\{-\mathbf{b}+\frac{1}{\|\mathbf{T}\|}(\mathbf{t a}) \mathbf{b}\right\} \frac{1}{\|\mathbf{b}\|}, \text { if } 0<\mathbf{a t}<\mathbf{t T}
\end{array}\right.
$$$$
\frac{\partial d_{e k}^{(i)}}{\partial \mathbf{x}_{i+1}}=\left\{\begin{array}{l}
0, \text { if } \mathbf{a t} \leq 0 \\
\left\{-\frac{1}{\|\mathbf{T}\|}(\mathbf{t a}) \mathbf{b}\right\} \frac{1}{\|\mathbf{b}\|}, \text { if } 0<\mathbf{a t}<\mathbf{t T}
\end{array}\right.
$$

\section{EXAMPLES}

A benchmark structural optimization problem, the cantilever beam optimization is explored in this section. For numerical simulation, we assume that the design material is homogeneous with unit Young's modulus and Poisson's ratio $v_{0}=0.3$. The planestress four-node elements are used to discretize the design domain. The design problems are solved with the maximum allowed material volume of $50 \%$ design domain volume, $f=0.5$.

\section{Cantilever beam design}

The cantilever beam problem is considered with design definitions given in Figure 3a. An analytical mesh of elements is employed. Figure $3 \mathrm{~b}$ shows the design by SIMP approach (using 99 lines Matlab code ${ }^{13}$ ), in which a sensitivity filter with a radius of 1.5 is employed to avoid the checker-board issue.

(a)

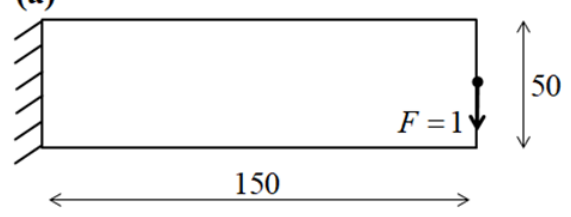

(b)

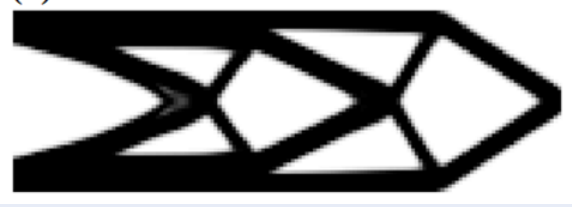

Figure 3: Cantilever beam design: (a) problem definitions, (b) optimizeddesign by SIMP ( $c=175.35)$.

To optimize the structure using the MPMVs approach, an initial design is predefined with 11 polygonal voids and 12 vertices for each polygonal void as presented in Figure 4a. The beam is optimized by searching the optimal positions of the polygonal voids. The optimized layout of MPMVs is plotted in Figure 4b, and the design is shown in Figure 4c.

It is worth mentioning that the MPMV approach significantly reduces the number of design variables compared with conventional approaches. The current design only uses 264 design variables that are much less than 7500 design variables by SIMP/ESO approach or 7701 design variables by level set approach. We observed that our overall optimum topology is in agreement with that by SIMP approach. Low-density elements inside structural boundaries may exist in the design by SIMP approach (see the middle part of the design in Figure 3b) but not exist in the design by MPMVs approach. The proposed approach produces a stiffer structure with $2.04 \%$ smaller compliance.

It is worth remarking that the structural boundaries are explicitly described by line segments of polygonal voids, hence the proposed method allows the ability to accurately capture structural boundaries to extract final designs. The computer-aided design (CAD) 
model can be obtained directly by keeping line segments on the structural boundaries while deleting unnecessary line segments. Hence, the proposed method allows capturing accurate structural boundaries in a cheap way compared with SIMP method, where the structure is implicitly described by the element density field that needs undergoing many steps of post-process for the final design.

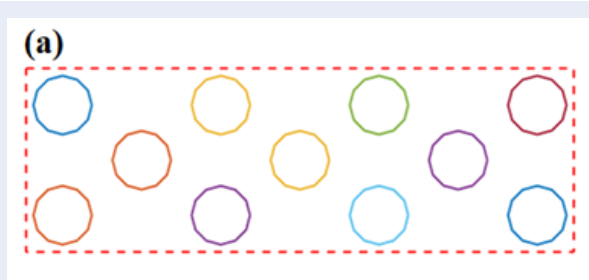

(b)

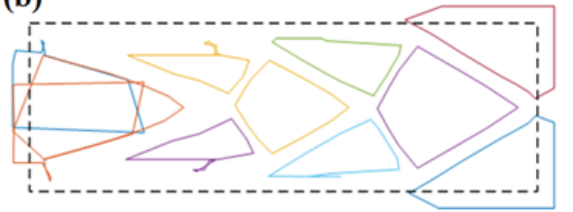

(c)

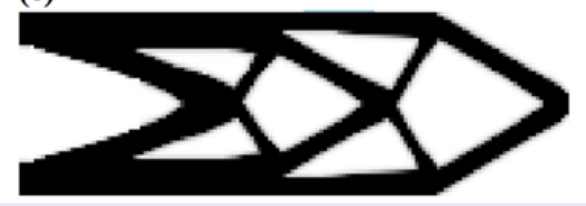

Figure 4: Cantilever beam design: (a) initial layout of MPMVs with 11 polygonal voids and 12 vertices for each polygonal void, (b) optimized layout of MPMVs, (c) optimized design (c=171.77)

\section{Mesh independency}

The above design problem is resolved with different meshes while retaining other design parameters. The optimum results are presented in Figure 5, in which Figure 5a plots the design with mesh 300x100 with compliance $c=171.91$ and Figure 5b plots the design with mesh $450 \times 150$ with compliance $c=171.99$. The convergence of all examples in this paper is obtained after 100 iterations.

Through three numerical examples in Figure 4c, Figure $5 \mathrm{a}$, and Figure $5 \mathrm{~b}$, it is observed as follows. The first observation is that the general optimum topologies are the same for three mesh cases: 150x50 elements, 300x100 elements, and 450x150 elements. The second observation is small differences in structural compliance: $c=171.77$ for the case of mesh $150 \times 50$ elements, $c=171.91$ for the case of mesh $300 \times 100$ elements, and $c=171.99$ for the case of mesh $450 \times 150$ elements. When a finer mesh is employed, the corresponding compliance increases, i.e., $0.08 \%$ when the number of mesh elements is increased from 150x50 elements to $300 \times 100$ elements and $0.05 \%$ when the number of mesh $300 \times 100$ elements is increased from 450x150 elements to elements. Another observation is that the checker-board issue does not appear in our design although we do not use any other techniques, i.e., filtering. These mean that optimized designs by the MPMVs-based approach depend on geometry parameters of MPMVs rather than the mesh size.

(a)

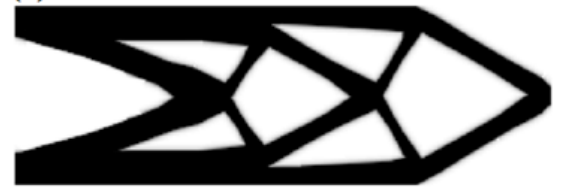

(b)

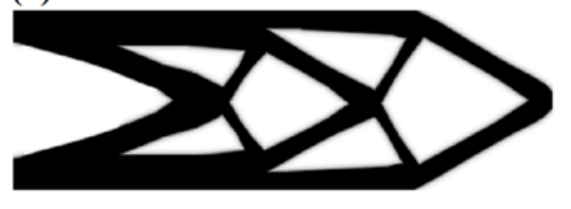

Figure 5: Mesh-independence: (a) result with mesh 300x100 (c=171.91), (b) result with mesh $450 \times 150$ ( $c=171.99$ ).

\section{CONCLUSION}

For the first time, an explicit topology optimization approach using MPMVs has been proposed for optimum structural designs. The MPMV-based approach allows mapping each polygonal void onto a fit subdomain instead of a full design domain. The density function of the elements located inside polygonal voids is realized by an exponential function that allows employing gradient-based optimization solvers. The proposed approach works effectively for two-dimensional structural optimization with a significant reduction of design variables. The filtering techniques or perimeter constraints are not necessary while still ensuring a mesh-independency solution. The extension of the current approach for threedimensional problems can be straightforward by replacing polygonal voids with polyhedral voids.

\section{ACKNOWLEDGMENTS}

This research is funded by Vietnam National Foundation for Science and Technology Development (NAFOSTED) under grant number 107.01-2019.317. 


\section{COMPETING INTERESTS}

The author(s) declare that they have no competing interests.

\section{REFERENCES}

1. Bendsøe MP. Optimal shape design as a material distribution problem, Struct. Optim. 1989;1:193-202. Available from: https://doi.org/10.1007/BF01650949.

2. Xie YM, Steven GP. A Simple Approach To Structural Optimization, Compurers Struct. 1993;49:885-896. Available from: https://doi.org/10.1016/0045-7949(93)90035-C.

3. Allaire G, Jouve F, Toader AM. A level-set method for shape optimization. Comptes Rendus Math. 2002;334:11251130. Available from: https://doi.org/10.1016/S1631-073X(02) 02412-3.

4. Saxena A. Topology design with negative masks using gradient search. Struct Multidiscip Optim. 2011;44:629-649. Available from: https://doi.org/10.1007/s00158-011-0649-4.

5. Guo X, Zhang W, Zhong W. Doing Topology Optimization EXplicitly and Geometrically-A New Moving Morphable Components Based Framework. J Appl Mech;81(2014):081009. Available from: https://doi.org/10.1115/1.4027609.

6. Norato JA, Bell BK, Tortorelli DA. A geometry projection method for continuum-based topology optimization with discrete elements. Comput Methods Appl Mech Eng. 2015;293:306-327. Available from: https://doi.org/10.1016/j. cma.2015.05.005
7. Hoang VN, Jang GW. Topology optimization using moving morphable bars for versatile thickness control. Comput Methods Appl Mech Eng. 2017;317:153-173. Available from: https: //doi.org/10.1016/j.cma.2016.12.004.

8. Hoang VN, Nguyen NL, Nguyen-Xuan H. Topology optimization of coated structure using moving morphable sandwich bars. Struct Multidiscip Optim. 2020;61:491-506. Available from: https://doi.org/10.1007/s00158-019-02370-z.

9. Wang X, Long K, Hoang VN, Hu P. An explicit optimization model for integrated layout design of planar multicomponent systems using moving morphable bars. Comput Methods Appl Mech Eng. 2018;342:46-70. Available from: https://doi.org/10.1016/j.cma.2018.07.032.

10. Hoang VN, Nguyen NL, Tran P, Qian M, Nguyen-Xuan H. Adaptive concurrent topology optimization of cellular composites for additive manufacturing. JOM. 2020;Available from: https: //doi.org/10.1007/s11837-020-04158-9.

11. Zhang W, Zhao L, Gao T, Cai S. Topology optimization with closed B-splines and Boolean operations. Comput Methods Appl Mech Eng. 2017;315:652-670. Available from: https:// doi.org/10.1016/j.cma.2016.11.015.

12. Zhang W, Chen J, Zhu X, Zhou J, Xue D, Lei X, et al. Explicit three dimensional topology optimization via Moving Morphable Void (MMV) approach. Comput Methods Appl Mech Eng. 2017;322:590-614. Available from: https://doi.org/10. 1016/j.cma.2017.05.002.

13. Sigmund O. A 99 line topology optimization code written in matlab. Struct Multidiscip Optim. 2001;21:120-127. Available from: https://doi.org/10.1007/s001580050176. 